\title{
LE CHORÉ
}

Julie PERRIN

\section{GRAPHIQUE}

TRAVERSÉ

PAR LA PHO

TOGRAPHIE

\section{À PROPOS DU TEMPS \\ DANS LA COMPOSITION: \\ RAINER, PAXTON ET \\ CHARMATZ}

Revue Ligeia, dossier "Photographie et danse » Debat Michelle (dir.), $n^{\circ} 113-114-$ 115-116, Paris, éd. Ligeia, janvier-juin 2012, pp. 74-83. 


\title{
LE CHORÉGRAPHIQUE
}

\author{
TRAVERSÉ PAR LA Julie PERRIN
}

\author{
PHOTOGRAPHIE
}

\author{
À PROPOS DU TEMPS DANS \\ LA COMPOSITION : RAINER, Revue $L$ Iigeia, dossier « Photographie \\ et danse » Debat Michelle

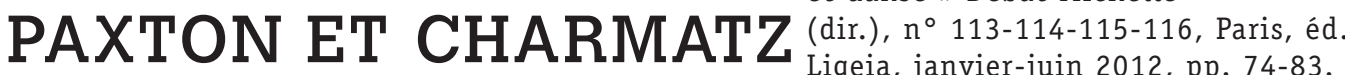

A l'ère de la reproduction mécanisée, la danse scénique et la photographie ont tissé des relations toujours plus riches et diversifiées. Il ne s'agit pas ici de retracer la longue histoire des liens entre pratiques photographiques et danse - histoire fondée, pour bonne part, sur une recherche quant à la saisie du mouvement. C'est une quête qui traverse l'histoire de la photographie, au-delà de ses rapports à la danse, depuis les travaux célèbre de la chronophotographie de Muybridge et Marey qui ont décomposé le mouvement animal ou humain. Il s'agit davantage de partir du champ de la danse et d'interroger l'influence du développement de la photographie sur l'esthétique de la danse moderne ou contemporaine. L'historienne de la danse Laurence Louppe a avancé, dans un article qui a fait date, que le réel est chargé en permanence d'un corps pris lui-même dans la construction des "images qui reproduisent son geste à l'infini »; le corps joue sans cesse avec ses doubles représentés, en une sorte de « contamination du corps par le mode de représentation iconographique ${ }^{1} »$. Laurence Louppe attire l'attention sur l'une des modalités de construction de la corporéité et du geste dansé. Un peu différemment, il s'agira ici de s'interroger sur un autre pan de cette influence de la photographie sur la danse : sur la façon dont une référence à la photographie s'introduit dans l'élaboration de la chorégraphie. Autrement dit, comment le modèle photographique fait-il intrusion dans la conception de la composition ? Ainsi, c'est emprunter la voie ouverte par la chercheuse en photographie Michelle Debat, qui demande : « Où et comment une certaine pensée de la danse croise-t-elle les mêmes questionnements conceptuels que la photographie, tels que le corps et le support, l'instant et le moment, l'image et le regard, l'espace et le temps ${ }^{2}$ ? ".

Parmi les divers usages de la photographie pour et par la danse, ce qu'il importe à chaque fois de démêler, c'est tout autant la (stratégie de) saisie du corps en mouvement par la photographie, que la façon dont la danse elle-même saisit la photographie. Façon dont elle s'en empare, l'intègre à son

\footnotetext{
1 Laurence Louppe, «Le Corps visible. La Photographie comme source iconographique de l'histoire de la danse moderne et contemporaine. Supports, genres, usages », in : L'Histoire de la danse, repères dans le cadre du diplôme d'Etat, Centre national de la danse, " Cahiers de la pédagogie », 2001, p. 48 et 49

2 Michelle Debat, L'impossible image. Photographie - Danse - Chorégraphie, La lettre volée, « essais », 2009, p. 15
} 
processus ou bien la destine à un prolongement ${ }^{3}$ (artistique, documentaire), mais aussi tout simplement la façon dont les danseurs ou chorégraphes la conçoivent, l'appréhendent. Qu'entendent-ils par " photographie »? Que recoupe l'usage du terme " photographie » dans leur discours, quel statut lui donnent-ils dans leurs pratiques chorégraphiques ? On verra que ce qui est en cause c'est tout autant l'image (une image photographique concrète, support ou sujet à création), qu'une idée de la photographie. Cela relève alors davantage de ce que Rosalind Krauss nomme « le photographique ${ }^{4}$ », c'est-à-dire la photographie comme objet théorique, à partir duquel s'ouvre un nouvel horizon de questionnement (esthétique, historique, sémiotique...).

Appliquée au champ de la composition en danse, la " photographie » devient ainsi prétexte à réflexion sur le temps. En effet, l'intrusion de la photographie et du photographique dans le champ du chorégraphique conduit en particulier à interroger la temporalité du mouvement et son rapport à la fluidité. Si la photographie est renvoyée au gel du mouvement, à l'instant qui vient soudain figer la pose, elle peut devenir métaphore tout autant que modèle pour explorer le flux. Rappelons que ce terme renvoie chez Laban à la qualité de propagation du mouvement dans le corps, avec d'un côté un flux libre qu'aucune tension musculaire n'entrave, de l'autre un flux lié ou contrôlé, propice à l'interruption du mouvement, à l'à-coup. Le photographique est l'occasion de réfléchir à la nature de la fluidité de la danse : comment s'organise la continuité, la progression du mouvement ? Il conviendra d'affiner cet imaginaire de la temporalité photographique. Mais dans un premier temps, notons qu'on est renvoyé avant tout à l'idée d'instantanéité, au déclic.

Aussi, l'intrusion du photographique dans la danse ne renvoie-t-elle sans doute pas à toutes les sortes d'arrêt du mouvement. Les poses longues donnant à éprouver la durée relèvent davantage de ce qu'on appellera des tableaux vivants. Résidu d'une pratique qui remonte au 18e siècle et allie théâtre et peinture, le tableau vivant instaure une interruption durable. La danse contemporaine le pratique abondamment, ainsi Ivana Müller pour While we were holding it together (2006) qui immobilise les interprètes en des tableaux que les récits successifs viennent réinterpréter ; ou Herman Diephuis pour D'après J. C. (2004) ouvertement inspiré de la peinture religieuse de la Renaissance. Lorsque Laure Bonicel fige en postures successives les interprètes de $N^{\circ} 11$ : le Bleu est à la mode cette année (2004), on pense aussi à des tableaux vivants (tableaux monochromes), bien qu'elle se réfère là explicitement aux photographies publicitaires de mode. C'est que l'histoire de l'iconographie (artistique ou non) témoigne de la circulation entre les arts et les médiums : la scène de genre composée de plusieurs personnages jouant un rôle bien défini, appartient tout autant à la peinture, à la photographie (en particulier au courant pictorialiste), qu'à la scène. Ce sont là des effets de transversalité entre peinture, photographie, théâtre ou danse. Dans l'art du tableau vivant, l'ordonnancement spatial importe tout autant que la fixation des postures de chacun. La durée de la scène conduit en effet à insister sur la structuration de l'espace, le rapport au cadre, le sens des distances entre les personnages... plutôt que sur la fluidité du mouvement soudain interrompue. Le tableau vivant permet surtout à la danse de renouer avec des modes du pictural ${ }^{5}$, plutôt qu'avec une pensée du photographique.

\section{Le photographique - arrêt sur l'acmé}

«Une grande part de la danse occidentale de laquelle nous sommes familiers peut être caractérisée par une certaine distribution de l'énergie : investissement maximal ou «attaque» en début de phrase, reprise à la fin, avec suspension de l'énergie quelque part au milieu. Ce qui signifie qu'une partie de la phrase - habituellement, celle qui est la plus arrêtée - focalise l'attention, enregistrant, comme une

\footnotetext{
3 Laurence Louppe a amorcé cette réflexion sur la photographie faisant « partie intégrante du processus chorégraphique, dont elle constitue à la fois une composante ou une extension », à partir des exemples de Catherine Contour ou de Benoît Lachambre, Saskia Hölbling, Xavier Le Roy collaborant avec le photographe Laurent Goldring. Laurence Louppe, « Danse-photographie. Pour une théorie des usages », art press, $\mathrm{n}^{\circ} 281$, juillet-août 2002, pp. 47-52

4 Rosalind Krauss, Le photographique. Pour une théorie des écarts, trad. Marc Bloch et Jean Kempf, Macula, Paris, 1990

5 Rappelons la connivence qu'ont connu la peinture et le ballet, la référence picturale étant par exemple omniprésente dans les Lettres sur la danse de Noverre (1760) qui avance que « les ballets sont des tableaux vivants » (Lettres XIII) et affirme : « un ballet est un tableau, la scène est la toile, les mouvements mécaniques des figurants sont les couleurs, leur physionomie est, si j'ose m'exprimer ainsi, le pinceau, l'ensemble et la vivacité des scènes, le choix de la musique, la décoration et le costume en font le coloris ; enfin, le compositeur est le peintre » (Lettre I).
} 
photographie, la suspension d'un moment d'acmé6. » La chorégraphe Yvonne Rainer, dans un texte désormais célèbre et généralement dénommé "L'intelligence est un muscle », pose ce qui constitue sans doute l'un des enjeux les plus discutés du photographique dans le champ de la danse. Il s'agit pour elle de qualifier les différentiels d'énergie dans l'exécution d'une danse, conduisant à des moments d'accélération ou ralentissement, voire de suspension, d'arrêt. La phrase dansée - autrement dit, la succession de quelques mouvement, ou encore, précise-t-elle, l'ensemble de la chorégraphie - répond traditionnellement à une loi de composition qui permet de ménager des temps forts reliés par des transitions. Ces temps forts sont considérés comme des moments photographiques.

Qui a fait l'expérience d'une " générale photo » (répétition d'une pièce destinée uniquement aux photographes professionnels) aura été frappé par la simultanéité du son du déclencheur des appareils : comme si la chorégraphie imposait en effet ses moments de capture photographique. Une telle remarque revient à retirer au photographe toute intention (ce qui est peut-être le sort de certaines photographies de presse) et à attribuer le surgissement de la photographie à l'objet même, qui, dans son arrêt fugace, provoque la capture instantanée. La danse se constituerait donc de poses successives. C'est précisément ce que vient interroger Yvonne Rainer et ce qu'elle souhaite désamorcer.

Avec Trio A (1966), Yvonne Rainer tente d'emprunter une autre voie pour la chorégraphie, qui ferait fi du photographique comme acmé : créer une danse anti-paroxystique, inventer une phrase sans tension, sans instants cruciaux, un continuum du mouvement fondé sur un travail d'égalisation et de désaccentuation tout à fait inhabituel sur la scène chorégraphique. Il s'agit de désamorcer un régime de plaisir ${ }^{7}$ convoque sur la scène, comme dans la salle, où le danseur exultait à canaliser l'attention du public. Conduire le regard du spectateur par la maîtrise d'une énergie exubérante, le fasciner, le transporter dans le plaisir de l'exhibitionnisme du geste dansé relèvent d'une esthétique (sans doute contraire à une forme d'éthique) dont Yvonne Rainer entend se défaire. La «photographie » dont parle Yvonne Rainer, c'est donc cet instantané à proscrire. Trio $A$ tentera cet impossible : devenir pure transition, chorégraphie de l'anti-photographie. Une danse qui échapperait au régime du photographique et même à la visibilité : « la danse est difficile à voir. (...) Cette difficulté intrinsèque doit être soulignée au point que la danse devienne presque imperceptible. (...) Mon Trio A traitait de la difficulté qu'il y a à «voir», à force de rendre implacablement évident le détail qui ne se répète pas et, ainsi, attirait l'attention sur la difficulté de cerner le matériau ${ }^{8}$. " Trio A rompt en effet la cohérence d'un mouvement construit par paliers qui permettait d'aboutir au surgissement d'une forme. Aucun dessin du geste ne semble pouvoir être cerné : des "mouvements discrets » et des sections créées de manière indépendante sont finalement additionnés sans logique particulière et reliés par une " dynamique piétonne ${ }^{9}$ » qui vient inonder l'ensemble... et noyer l'attention du spectateur.

Si Yvonne Rainer croit en la capacité de la danse d'annuler le principe de saisie du visible, elle s'attèle également à neutraliser la charge émotive - celle portée par l'interprète et susceptible de transpirer jusque vers le spectateur. Neutraliser la disposition émotive du spectateur revient à désamorcer le déclic (photographique) qui peut s'opérer chez le regardeur, lorsqu'il devient sujet d'une expérience qui le saisit. Si le drame disparaît, si l'interprète camoufle tout investissement émotif, une relation plus distante pourra s'instaurer avec le public qui, loin de toute complicité, atteindra peut-être également cette forme de neutralité : c'est du moins l'hypothèse de travail de la chorégraphe.

Ainsi Yvonne Rainer s'attaque au photographique par tous les bords. Du côté de " la cible », on l'a vu, ce que Barthes appelle "le Spectrum de la photographie », c'est-à-dire " celui ou cela qui est photographié ${ }^{10} »$; aussi bien que du côté du Spectator qui, dans notre cas présent, est tout autant un

6 Yvonne Rainer, « De la presque survie de quelques tendances «minimalistes», repérables dans l'activité quantitativement minimale de la danse parmi la pléthore, ou analyse de Trio $A$ »(1966), trad. adaptée de Christophe Wavelet in Life/Forms (vita/formae), catalogue d'exposition, Musée Fesch, Ajaccio, 1999, p. 99, texte plus fréquemment mentionné sous le nom de « The Mind is a Muscle » (L'intelligence est un muscle), publié dans Yvonne Rainer, Work 1961-73, The Press of the Nova Scotia College of Art and Design, Halifax, New York University Press, New York, 1974, pp. 63-69.

7 C'est un régime de plaisir que Steve Paxton, Yvonne Rainer et la critique Jill Johnston qualifient d'orgasmique. Cf Julie Perrin, « L'obscénité chorégraphique », in Obscène, Obscénités, Actes du colloque organisé par le CREM de l'université Paul Verlaine à Metz en 2006, (Steven Bernas, Jamil Dakhlia, dir.), L'Harmattan, « champs visuels », 2008, pp. 73-82. Egalement disponible sur : http://www. danse.univ-paris8.fr

8 Yvonne Rainer, «De la presque survie de quelques tendances «minimalistes»», art. cit., trad. de Christophe Wavelet, p. 102

9 Rainer Yvonne, « Profile: Interview by Lyn Blumenthal » (1984), A Woman Who... Essays, Interviews, Scripts, The Johns Hopkins University Press, « Art + Performance », Baltimore (Maryland), 1999, p. 62 et 64.

10 Roland Barthes, La Chambre Claire (1980), CEuvres complètes V, Seuil, Paris, 2002, p. 795 
photographe (Operator) imaginaire, métaphorique. Si le spectateur, comme le photographe barthésien, « est essentiellement témoin de sa propre subjectivité, c'est-à-dire de la façon dont il se pose, lui, comme sujet en face d'un objet ${ }^{11}$ ", face à Trio $A$ il est renvoyé à l'impossibilité d'un positionnement stable, renvoyé à l'impossibilité de cerner les contours de l'objet. De plus, le rejet du pathos et de la complicité de part et d'autre revient à tenter d'empêcher le jeu de l'affect si cher à Barthes dans sa définition dernière de la photographie ${ }^{12}$, où le spectateur existe dans l'émoi, est révélé par la photographie qui l'anime, le pique : c'est le fameux punctum. Rainer fait donc en quelque sorte le vœu de $s^{\prime}$ en tenir au seul studium, ce " champ très vaste du désir nonchalant ${ }^{13}$ », lieu d'un contrat docile entre l'auteur et son spectateur. Dans ce consentement attendu par Rainer, la gêne produite par la nature inhabituelle et insaisissable de la danse fait pourtant affleurer le sujet spectateur : un spectateur qui ne s'annule pas dans l'absorption dans l'objet de sa contemplation, mais est sollicité de manière réflexive sur son activité même.

La définition du photographique chez Yvonne Rainer relève d'une méfiance que d'autres chorégraphes ont pu partager mais qui donne lieu ici à un positionnement extrême que la chorégraphe ne pourra strictement tenir que pour cette œuvre-manifeste. Dans les débuts de la danse moderne pourtant si souvent enclins à la photographie, on a pu voir une Mary Wigman s'inquiéter que le photographe mette trop de lui-même, mais se réjouir aussi que Charlotte Rudolph, qui deviendra sa photographe de prédilection, ait su saisir le sens de sa danse ${ }^{14}$. C'est peut-être justement parce que la photographe a développé une réflexion sur le flux, la pause, les temps forts, qu'elle parvient à satisfaire la chorégraphe. Charlotte Rudolph s'efforce en effet à "s'accorder entièrement avec le rythme», à développer "un sens de la danse », afin d'en " capter le moment juste ${ }^{15}$ ». Surtout, sa pratique photographique s'appuie sur une analyse de la danse divisée en temps forts et moments de transition. On retrouve donc l'analyse du flux ou du phrasé qui hante les rapports entre danse et photographie. " Par temps fort, explique Charlotte Rudolph, j'entends par exemple : moment de tension extrême, moment de détente extrême, moment de suspension. De moment de transition, je qualifie par exemple un moment où l'on passe d'un mouvement à l'autre, un moment choisi au hasard lors de l'exécution d'un mouvement, un moment extrait d'un rythme bref. Par comparaison, les temps forts sont plus faciles à photographier, excepté les sauts, tours et autres mouvements de ce genre. Les moments de transition nécessitent encore plus de synchroni ${ }^{16}$. » Mais à l'opposé de la définition du photographique que l'on connaîtra chez Yvonne Rainer, la photographe Charlotte Rudolph s'applique à saisir la danse dans ses moments de transition: car c'est là que se révèle « la touche personnelle ${ }^{17}$ » du danseur (surtout chez les danseurs doués de lyrisme). La photographie n'a donc que faire ici de l'acmé du mouvement. De son point de vue de chorégraphe, Odile Duboc à sa manière réhabilite également la fluidité, la continuité, la progression, accordant néanmoins au spectateur la liberté d'un instantané photographique. Cet instantané ne coïncide pas nécessairement avec les suspensions et apnées chères à la musicalité dubocienne, mais il naît de l'émotion du spectateur. Tout autant photographique que chorégraphique, l'instantané n'est pas imposé par la danse. "Le spectateur ne peut-il pas lui-même prendre au vol et fixer dans sa mémoire ces instants d'émotions ? Lorsque la danse existe sans s'imposer, le spectateur, si la chorégraphie le lui permet, se sentira alors investi d'un nouveau rôle actif : celui de photographe de l'instantané ${ }^{18}$. » Mais tous les photographes, loin s'en faut, ne soutiennent pas la position de Charlotte Rudolph. Certains préfèrent en effet faire coïncider l'acmé dansée et l'instant photographique, telle Barbara Morgan, fidèle photographe de Martha Graham : "Chaque danse a ses pics d'intensité émotionnelle; moments où la danse «parle» au public. Ce sont des moments où la forme de la danse est dans le plus proche unisson avec la compulsion originelle qui lui a donné naissance. (...) La photographie de danse traite des moments nets daction expressive ${ }^{19}$. »Ce sont ces moments, selon Barbara

11 Roland Barthes, «Sur la photographie » (février 1980), EEuvres complètes V, Seuil, Paris, 2002, p. 933

12 Dans La chambre claire, op. cit. Pour une étude sur l'évolution des conceptions de la photographie chez Barthes, voir Bernard Comment, Roland Barthes, vers le neutre, Christian Bourgois, Paris, 1991

13 Roland Barthes, La Chambre Claire, op. cit., p. 810

14 Voir Hedwig Müller, «L'expression Mary Wigman », in Photographies, n 7, mai 1985, pp. 64-69

15 Charlotte Rudolf, «La photographie de danse » (1929), trad. inédite d'Axelle Locatelli de " Tanzphotographie », in Schrifttanz, Deutsche Gesellschaft für Schrifttanz, mai 1929

16 Idem

17 Idem

18 Odile Duboc, «L'instantané chorégraphique », in Lorina Niclas (dir.), La danse, naissance d'un mouvement de pensée ou Le complexe de Cunningham, Armand Colin, Biennale Nationale de danse du Val-de-Marne, Paris, 1989, p. 175

19 Barbara Morgan, Martha Graham : sixteen dances in photographs, Duell, Sloan and Pearce, New York, 1941, non pag. (C'est nous qui traduisons) 
Morgan, que retient la mémoire dans le continuum de l'espace-temps, ces moments que la photo doit alors mémoriser et transmettre.

\section{Acmé, mémoire et histoire}

La question de la mémoire est en réalité centrale dans ce débat sur la composition s'articulant avec une pensée du photographique et plus particulièrement de l'instantané. Trio $A$, en refusant l'interruption du flux, interdit toute pause du regard. L'attention du spectateur, saisie dans ce flux, jamais ne peut s'arrêter le temps de mémoriser ce qui vient de se produire. Sans répit, la danse impose son rythme tranquille et nonchalant au risque de produire son propre effacement. Au final il ne reste, écrit Sally Banes, qu'une " tresse [ligne de mouvements multiples simultanés] si étroitement enserrée par la monotonie qu'elle se transforme dans le regard et la mémoire du spectateur en une unique figure, longue et indivisible ${ }^{20}$. " C'est que Trio $A$ refuse aussi la répétition, autre forme possible d'une vue rétrospective sur ce qui vient de se produire, autre manière d'en retenir une image.

Comment dès lors photographier Trio $A$ ? L'on rêverait de voir quel regard auraient porté sur cette danse Charlotte Rudolph (1896-1983) ou Barbara Morgan (1900-1992) ; comment l'une aurait épousé sa qualité de transition (pourtant dénuée de lyrisme) ; comment l'autre se serait confrontée à l'absence de climax, de drame, d'intensité émotionnelle : qu'aurait-elle pu saisir ? Quels gestes seraient venus se fixer « de manière très nette dans [sa] mémoire [pour venir symboliser] la danse toute entière ${ }^{21}$ » ? Mais voilà, ni l'une ni l'autre n'a tourné son objectif vers cette danse. Grande est alors la tentation de se pencher vers les photographies existantes de Trio A. C'est ce qu'a fait Carrie Lambert-Beatty, dans l'un des livres les plus remarquables sur cette chorégraphie : Being Watched. Yvonne Rainer and the $1960 s^{22}$. L'auteure y examine ce qu'elle analyse comme un écart stupéfiant : les photographies de Trio A donnerait à voir une danse spectaculaire, une physicalité expansive proche de l'exhibition dramatique et athlétique absolument contraires, donc, aux intentions de la danse ${ }^{23}$. Ces affirmations pèchent sans doute par un manque d'observation plus fine du mouvement représenté, qui permettrait d'analyser non seulement la cinématique du mouvement (sa forme, sa géométrie), mais également sa dynamique caractérisant l'investissement énergétique du danseur. En associant cinématique et dynamique, on peut lire ce qui advient et non plus seulement ce qui se fige sur la photographie. Pourtant Carrie Lambert-Beatty $n^{\prime}$ en conclut pas à l'incompatibilité fondamentale entre cette danse et sa saisie photographique ${ }^{24}$. D'une manière plus intéressante, elle en déduit le flux paradoxal de Trio $A$ : sa nature, on l'a vu, tout à la fois segmentée (les petites sections mises bout à bout) et unifiée. Comme si la photographie venait révéler la part secrète de Trio $A$, la part cachée sous le continuum de la dynamique piétonne.« La continuité paradoxale de Trio $A$ incorpore une interruption quasi-photographique, par exemple des traces performées de danses passées ${ }^{25}$ ». En effet, comme l'avait déjà noté Sally Banes, Yvonne Rainer joue avec l'histoire de la danse, et tout particulièrement celle du ballet classique, introduisant des citations que le continuum et l'exécution nonchalante effacent dans le même temps. Arabesque grahamienne, port de bras et en-dehors classiques, figure de danseur noble, référence kathakali, faune de Nijinski peut-être ${ }^{26}$. Aussi, le projet est-il tendu entre d'une part la volonté de neutraliser toute possibilité d'anticiper sur le mouvement à venir, comme de mémoriser celui qui vient d'être effectué, et d'autre part l'intrusion de citations masquées, susceptibles pourtant de focaliser l'attention un instant. En sollicitant les images de la danse et la mémoire, Yvonne Rainer prend le risque de faillir à son projet

20 Sally Banes, Terpsichore en baskets. Post-modern dance, Chiron / Centre national de la danse, Paris, 2002, trad. Denise Luccioni (Terpsichore in Sneakers : Post-Modern Dance, Houghton Mifflin Company, Boston, 1980), p. 98

21 Barbara Morgan, op. cit. : «Quels sont les moments significatifs pour le photographe ; et comment faut-il les choisir ? Je fais ma sélection en regardant un grand nombre de représentations et répétitions - notant particulièrement ce qui est abandonné. Après cette absorption aussi forte que possible, je trouve que certains gestes demeurent fixés de manière très nette dans ma mémoire, et viennent symboliser la danse tout entière. » (C'est nous qui traduisons)

22 Carrie Lambert-Beatty, Being Watched. Yvonne Rainer and the 1960s, MIT Press, coll. October Book, Cambridge, London, 2008.

23 Ibid., p. 127, puis 132 et sq..

24 Malheureusement, l'auteur en vient à conclure que parce que Trio A est tout transition, il serait tout autant entièrement acmé : autrement dit un moment continu de photogénie. (Ibid., p. 164) C'est là un retournement fâcheux dans son analyse du mouvement - de son flux, de sa dynamique -, mais aussi une définition réductrice de la photographie de danse (cf supra).

25 Ibid., p. 157 (C'est nous qui traduisons)

26 Yvonne Rainer n'a que tardivement (et vaguement) révélé cet aspect de Trio A. Son texte « L'intelligence est un muscle » n'en fait pas mention. 
de continuum radical, mais affirme dans le même temps sa tentative d'effacement de l'histoire ${ }^{27}$. Faire table rase ? Pas exactement. Plutôt réintégrer l'histoire mais en annulant son impact, sa puissance : tel est l'effet de ce flux paradoxal.

\section{La photographie comme réservoir de gestes}

Ainsi, le refus du photographique chez Yvonne Rainer s'accommode au passage de différentes photographies empruntées à l'histoire de la danse. En effet, la chorégraphe avoue avoir dévoré à cette époque des livres d'images de danse, et fréquenté assidûment le fonds photographique de la New York Public Library ${ }^{28}$. Selon un procédé souvent pratiqué par le Judson Dance Theater, Rainer emprunte et fait siens des gestes observés sur des photographies.

Steve Paxton a adopté ce principe de composition à plusieurs reprises à l'époque du Judson. Pour Proxy (1961), deux partitions de mouvements issues de photographies constituent les parties centrales de ce trio en quatre sections. Ces photographies présentent des gens qui marchent ou font du sport, mais aussi des images de dessins animés ou des publicités pour le voyage. Elles sont collées par Paxton sur un grand morceau de papier disposé en coulisse, de sorte que les danseurs puissent s'y référer pendant l'exécution de leur danse ${ }^{29}$. Steve Paxton reprend le principe en 1963 pour English, lors du quatrième concert de danse à la Judson Church. Cette pièce pour onze danseurs repose en grande partie sur des marches tranquilles du groupe, quelques séquences de mimes d'actions quotidiennes, tandis que les uns ou les autres agissent selon les indications tirées de photographies de groupe représentant des sportifs. Configurations de jeux, sauts athlétiques, les interprètes passent d'une position à l'autre, reconfigurant le groupe progressivement ${ }^{30}$. La photographie est donc ici utilisée comme support à la création du geste. Que Paxton s'appuie justement sur des sujets en mouvement participe à la mise en place d'une gestuelle détachée de la production du chorégraphe. En effet, l'un des enjeux de ce procédé est politique : il s'agit de sortir de la relation traditionnelle et hiérarchique entre danseur et chorégraphe, entre maître et élève. Dans ces deux pièces, Steve Paxton laisse en effet aux danseurs la liberté de construire les transitions entre deux poses, s'attachant simplement à l'analyse précise de la posture photographiée. Il est en quelque sorte chorégraphe par procuration - c'est le sens du titre Proxy - et d'autant plus quand l'interprète est invité à choisir où démarrer sa séquence dans la boucle des photographies affichées. La seule question soulevée, déclare-t-i ${ }^{31}$, concerne la façon d'interpréter la transmission photographique du geste : faut-il considérer la photographie en miroir (de même que le professeur de danse lorsqu'il fait face aux élèves) et donc coordonner la jambe gauche du sujet représenté avec la jambe droite du danseur ? Ou au contraire reproduire le même mouvement ? Question technique donc, qui pose le problème de la lecture de la photographie dès lors qu'elle constitue un programme d'actions.

C'est sur un principe similaire que Boris Charmatz engage en 2009 avec des étudiants du Hüz Tanz à Berlin un projet pédagogique nommé All Cunningham qui sera réitéré dans d'autres cadres ${ }^{32}$, jusqu'à devenir une pièce pour la scène dénommée Flip book, interprétée par des danseurs professionnels, puis une pièce destinée à d'anciens danseurs de la compagnie Merce Cunningham, sous le nom de 50 ans de dans $^{33}$. Boris Charmatz choisit pour support à ce projet la monographie rédigée par David Vaughan ${ }^{34}$, plus précisément, la succession des photographies qu'elle présente. 0n y trouve essentiellement des photographies illustrant les nombreuses pièces signées par le chorégraphe, mais également des photographies qui documentent son enfance (des photos de famille) et ses débuts (des pièces de Graham, par exemple) ou des portraits (du chorégraphe, de collaborateurs ou de la compagnie). Thèmes hétéro-

27 D'une manière semblable, Bernard Comment souligne combien la tentation du Neutre chez Barthes, le refus du pathos et de l'emphase a quelque chose à voir avec une forme de malaise historique qu'il éprouve. 0p. cit., p. 9

28 Selon un témoignage à Carrie Lambert-Beatty, op. cit., p. 48

29 On trouve une description détaillée de cette danse dans Sally Banes, Democracy's Body. Judson Dance Theater, 1962-1964, Duke University Press, Durham London 1995 (1ère éd. UMI Research Press, Michigan, 1980), pp. 58-60

30 Ibid., p. $97-98$

31 Ibid., p. 59

32 En particulier avec des étudiants de l'université de Rennes, ville où Boris Charmatz dirige le Musée de la danse, et au département danse de l'Université Paris 8 : le projet s'appelle alors Tout Cunningham ou Roman photo.

33 Pour cette analyse, nous nous appuyons essentiellement sur 50 ans de danse, 2009.

34 David Vaughan, Merce Cunningham. Un demi-siècle de danse, Plume / Librairie de la danse, 1997, trad. Denise Luccioni de : Merce Cunningham: Fifty years, Ed. Aperture, 1997 
clites, donc, mais présentant toujours un sujet humain dont le danseur peut s'emparer comme prétexte à mise en mouvement, ou prétexte aux poses successives. Boris Charmatz semble renouer avec des procédés de composition propres au Judson, dont Robert Ellis Dunn avait ouvert la voie. Dans l'atelier de Dunn, il s'agissait en effet de sortir d'un mode de composition organique dont le modèle prégnant demeure celui de la croissance de la nature. Les danseurs y expérimentaient procédés aléatoires, collages (et références dadaïstes), structures empruntées à des partitions de musique contemporaine (selon le principe de Cage), explorations de programmes d'action ou scores tels qu'Anna Halprin les avait aussi pratiqués... S'il est tentant de rapprocher Proxy et English de 50 ans de danse, l'enjeu de la pièce de Boris Charmatz semble quelque peu différent.

Comme chez Steve Paxton, il s'agit à première vue de se saisir de photographies, d'en déplacer l'usage purement documentaire et descriptif, pour en extraire la dimension performative. Pourtant, chez Boris Charmatz, se greffe une préoccupation historique absente chez Steve Paxton. Ce dernier ne cherche en rien à reconstituer un événement (un match, par exemple), ni à construire l'unité thématique d'un projet (le sport ? mais tout aussi bien le mouvement quotidien). La nature documentaire ou mémorielle de la photographie est peu pertinente. Chez Boris Charmatz, le sujet de la photographie est déterminant et donne d'ailleurs à la pièce ses titres successifs. D'un côté le chorégraphe se défend d'une préoccupation historique ${ }^{35}$ et semble donc s'éloigner d'un usage documentaire des photographies, de l'autre il déclare trouver par ce projet une proximité avec le chorégraphe : dans le procédé de composition (« l'enchaînement des images renvoyait au processus de composition des pièces de Cunningham $\left.{ }^{36} »\right)$, dans la ressemblance obtenue (« une chorégraphie qui suivrait le fil de ces photos ressemblerait sans doute elle aussi à du Cunningham $\left.{ }^{37} »\right)$. Autrement dit, le lien avec le référent photographique n'est pas purement cinétique, il s'agit bien de constituer un tout dont le référent ultime - Cunningham - est primordial. L'évolution des titres est significative de cette indécision : du Tout Cunningham qui fantasme l'exhaustivité de l'œuvre (et de l'homme ?), on passe à Roman photo qui avoue son rapport à la fiction, pour finir avec Flip book ou 50 ans de danse qui signalent précisément le livre de Vaughan. On est passé du monument (Cunningham), aux documents (les photographies du livre). Une question demeure : quel statut est accordé à la photographie dans l'usage et le discours du chorégraphe?

\section{La photographie : index ou bien fiction histo- rique?}

La photographie est d'abord réservoir de mouvements. Le projet se monte en cinq jours en moyenne, rapidité qui signale l'impossibilité à décrypter en détail les quelque trois cents photos. 0n y extrait donc avant tout une information cinétique qui devient défi technique pour le danseur (comment effectuer telle ou telle pose ?). La précision ne sera sans doute pas toujours de mise : c'est la production rapide du geste qui est en jeu, laissant de côté l'esthétique photographique (le travail de l'image, le point de vue du photographe, le style). La photo s'annule comme médium, le référent (la réalité représentée) tente d'adhérer au présent. Pourtant, la photographie persiste comme image gelée, en posant un défi à la composition. Boris Charmatz insiste moins que Steve Paxton sur le travail de la transition et interprète la photographie dans sa dimension arrêtée : il ne s'agit pas de retrouver le flux d'une danse mais de donner à voir des images une à une. Enfin, bien que Charmatz reconnaisse faire du «faux Cunningham », il ne parvient pas à se départir de la croyance en la ressemblance : la photographie, sur le mode de l'index, de l'empreinte, est rapportée à un objet de vérité. Le discours de Charmatz oscille donc entre vérité et fiction : « je pense que si nous y parvenons, c'est (...) réellement d'une pièce de Cunningham qu'il s'agirait, un event méta-Cunninghamien avec un aperçu de toute sa vie et son œuvre $^{38}$ ». Ainsi, non seulement l'image est devenue l'œuvre, mais l'interprétation ou la performance de l'image peut prétendre à retrouver l'œuvre.

35 A plusieurs reprises dans l'Entretien avec Gilles Amalvi, Programme du festival d'automne, juin 2009.

36 Idem

37 Idem

38 Texte du programme de Flip book, 2009. Sur le rapport entre document et fiction, voir Jean-Claude Moineau, « De la photographie comme opérateur critique à la photographie comme opérateur d'art », Ligeia, n 49-50-51-52, janv-juin 2004 
A l'exact inverse de Rainer qui masque la citation historique, ce projet égrène des images que le spectateur est susceptible de reconnaître. On est face à deux formes de désir et de deuil, pour reprendre la métaphore romantique de Barthes : qu'en est-il du « ça a été39 » de la photographie de danse ? Dans la logique de Barthes, la photo citée renverrait tout autant à la réalité de la danse qu'à son caractère irrémédiablement passé. Rainer redouble la mort portée par la photographie, en effaçant subtilement son impact (et la souffrance de la disparition). Boris Charmatz, lui, met en évidence le « ça a été » très joyeusement, c'est-à-dire sans nulle nostalgie. Mais il ne s'en tient pas là, car il entreprend aussi, paradoxalement, un projet de remémoration (proustienne), sans s'en donner les moyens pourtant. N'est-il pas question de faire fi du «ça a été » en restituant ce qui est aboli, ce « tout Cunningham »? Partant, il opère une autre forme de mise à mort, malgré lui. Par insuffisance historique, par défaut de lecture des photos également.

Si le collage a pu faire partie de ses modes de composition, Cunningham n'a jamais, à notre connaissance, fait appel à des photographies, mais surtout son usage de l'aléatoire est méthodique, ainsi que défini par Cage, et en rien " sauvage ${ }^{40}$ » ni même collectif. Si la pose fait partie de nombreuses chorégraphies, Cunningham est particulièrement sensible à l'équilibre qui s'opère entre flux et positions : l'enjeu est de préserver la clarté des positions, certes, mais à l'intérieur du flux ; si on perd le rythme, l'énergie, " on tombe dans la décoration ${ }^{41}$ », dit-il. Boris Charmatz met à mal le flux cunninghamien par des accents, accélérations, et ignore cette qualité particulière de tranquillité : en mettant les interprète dans un " état d'urgence » qui les conduit par une " énergie explosive ${ }^{42}$ » dans une course de pose en pose, il n'atteint jamais cette vibration de l'immobilité du corps en recherche d'un équilibre posé, pourtant si visible sur les photographies. Et l'espace en négatif entre les danseurs, si cher au chorégraphe lors du suspens du mouvement (comme dans Pictures, 1984), ne parvient pas ici à transparaître. Question de temporalité, donc, mais aussi question d'espace. Curieusement, le travail à partir de la photographie conduit à une occupation du plateau frontale et centrée, comme si soudain le cadre et point de vue photographiques prenaient le pas sur l'espace scénique référentiel. De plus, $l^{\prime}$ emplacement sur la page du livre oriente les $\operatorname{choix}^{43}$. Ou encore, comme il n'y a que très rarement chevauchement entre photographies, l'attention n'est focalisée qu'en un seul point du plateau, au lieu de la dispersion de l'attention si caractéristique. Enfin, ce qui fait que cette pièce ne ressemblera jamais à du Merce Cunningham, c'est bien le motif de leur danse : on y lit explicitement une organisation des uns par rapport aux autres, une attente avant de rejoindre l'autre afin de compléter l'image. Les mobiles sont évidents et contraires à cette indépendance si forte du danseur parmi le groupe, dans la plupart des pièces de Cunningham.

Autrement dit, le pari de la photographie tel qu'il a été pris dans 50 ans de danse a conduit à faire émerger au mieux une réminiscence de l'image pour le spectateur familier du livre de Vaughan. C'est bien l'image photographique qui a pu être remémorée, mais beaucoup moins les danses elles-mêmes et encore moins LA danse de Cunningham (projet voué d'avance à l'échec, dès qu'on observe l'évolution de son œuvre et de sa technique). Certes, on pourra reconnaître lors de quelques transitions plus travaillées un duo de Rainforest, une traversée lente en arabesques successives de Walkaround time ou un pas de gigue venu de Roaratorio, mais on verra poindre surtout une forme d'ironie bien absente des photographies : ironie de certains pas trop marqués ou de fentes réalisées lourdement, exagération d'une gesticulation frénétique en référence lointaine au rythme si particulier de Cunningham-interprète, parodie enfin en ces justaucorps à paillettes ou couleurs vives si différents de la recherche menée par les plasticiens créateurs de costumes ${ }^{44}$. Cela correspond-il à un choix assumé d'une poétique de la reprise, sur le mode d'une distanciation drôle et joyeuse ? Pour finir, dans son dispositif même, la prétendue libération de l'auteur grâce à l'usage de la photographie est presque contredite par la présence de Boris Charmatz, dans la fosse, mais bien visible, qui tourne les pages du livre de Vaughan, comme si le procédé ne pouvait se donner à lire de lui-même. Boris Charmatz, comme Cunningham

39 Roland Barthes, La chambre claire, op. cit., p. 851

40 Entretien avec Gilles Amalvi, art. cit. : «l'aléatoire fait partie intégrante de la démarche, particulièrement en ceci : nous nous mettons devant les images, et choisissons de manière assez «sauvage»». Idem

41 Merce Cunningham, Le Danseur et la danse. Entretiens ave Jacqueline Lesschaeve, Pierre Belfond, " Entretiens » 1988 (1ère éd. 1980), p. 139

42 Selon Anne-Karine Lescop, " Des photographies comme partition. Entretien avec Anne-Karine Lescop autour d'All Cunningham », in Repères. Cahier de danse, Biennale nationale de danse du Val-de-Marne, $n^{\circ} 23$, avril 2009, p. 28

43 Idem. Une image en bas de page invite à se mettre à l'avant-scène.

44 Voir Julie Perrin, «Le costume Cunningham : l'académique pris entre sculpture et peinture », Repères. Cahier de danse, Biennale nationale de danse du Val-de-Marne, $n^{\circ} 27$, avril 2011, p. 22-25 
d'ailleurs, ne tient pas jusqu'au bout le pari politique d'une remise en question des rôles au sein d'une compagnie.

Au fond, le projet peine, semble-t-il, à se définir. Car si l'usage de la photographie comme générateur de gestes est limpide et efficace (et très fécond dans un cadre pédagogique), la question du flux ne semble pas résolue et surtout le rapport de la photographie à la mémoire et à l'histoire donne lieu à une série de contradictions dans la pratique comme dans le discours.

\section{Temps, image et regard}

Le modèle photographique fait donc irruption dans la conception de la composition en danse de maintes façons, révélant des usages à chaque fois spécifiques et des conceptions de la photographie qui ne sont pas toujours explicitées par les artistes, mais que l'examen de leurs pratiques dévoile. On l'a vu, le photographique est le lieu d'une interrogation sur l'acmé, une réflexion sur le temps et le flux. La photographie, l'image, devient quant à elle matière à inventer le mouvement et à composer de nouvelles danses. Manière de repenser aussi le geste chorégraphique et la position de l'auteur. Moyen enfin pour une réflexion historique.

La réflexion sur la photographie permet d'ouvrir une lecture possible du chorégraphique, dans sa dimension temporelle. Elle permettrait aussi, mais ce serait l'objet d'une autre réflexion, de creuser une lecture spatiale de la composition en danse : de quelle façon la danse puise-t-elle dans une culture de l'image qui informe ses choix d'organisation spatiale ? Où l'on verrait combien parfois le cadre de scène semble coïncider avec un cadre photographique. Ou combien la danse peut frayer avec une fabrique de l'image - par la construction des plans, du point de vue, du hors-champ, par la disposition des couleurs et des contrastes. Plus étonnant, la danse a pu aussi interroger le modèle photographique à partir non de son résultat mais de son procédé. C'est bien l'Operator que va mettre en scène Vincent Thomasset, c'est-à-dire celui qui fait - le photographe - celui dont Barthes ne parle jamais car il n'en a pas l'expérience. Dans ses ateliers initiés en 2009 et intitulés « No camera » ou « Comment prendre des photos sans appareil photo ? ", l'artiste chorégraphique fait travailler le geste du photographe même : corps tendu par la concentration du regard, corps soudain en arrêt face à l'objet de sa photographie imaginaire, corps replié vers un détail à atteindre, errance à la recherche d'un sujet à saisir. Là encore, il a des pauses, dont la durée est exacerbée et qui se maintiennent bien plus que le temps d'un déclic imaginaire. Des pauses qui viennent surligner une chorégraphie du regard.

Julie Perrin

Pour citer cet article : Julie Perrin, "Le chorégraphique traversé par la photographie. À propos du temps dans la composition: Rainer, Paxton et Charmatz», revue Ligeia, dossier "Photographie et danse » Debat Michelle (dir.), n 113-114-115-116, Paris, éd. Ligeia, janvier-juin 2012, pp. 74-83.

Cité d'après la version électronique publiée sur le site Paris 8 Danse : www.danse.univ-paris8.fr 